\title{
The superiority of 256-slice spiral computed tomography angiography for preoperative evaluation of surrounding arteries in patients with gastric cancer
}

\author{
This article was published in the following Dove Press journal: \\ OncoTargets and Therapy \\ 16 February 2017 \\ Number of times this article has been viewed
}

\section{Deqing Wu \\ Linyong Zhao \\ Ying Liu \\ Junjiang Wang \\ Weixian $\mathrm{Hu}$ \\ Xingyu Feng \\ Zejian Lv \\ Yong $\mathrm{Li}$ \\ Xueqing Yao}

Department of General Surgery, Guangdong General Hospital and Guangdong Academy of Medical Sciences, Guangzhou, People's Republic of China
Correspondence: Xueqing Yao Department of General Surgery, Guangdong General Hospital and Guangdong Academy of Medical Sciences, Guangzhou, 51 0080, People's Republic of China

Email yjb92I I@2Icn.com
Objective: To evaluate the utilization of 256-slice spiral computed tomography (CT) angiography in preoperative assessment of perigastric vascular anatomy in patients with gastric cancer.

Methods: In this study, 80 gastric cancer patients were included. The medical procedure of 256-slice spiral CT angiography was performed on each of these patients consecutively. Thereafter, these patients were subjected to surgical treatment in our hospital. The techniques of volume rendering (VR) and maximum intensity projection (MIP) were used to image reconstruction of arteries around the stomach.

Results: Both VR and MIP were applied to reconstruct the images of perigastric arteries. The results indicated that VR imaging was inferior to MIP in determining the variant small artery anatomy around the greater curvature and fundus. The respective rates of imaging produced by VR and MIP for left gastroepiploic artery, short gastric artery, and posterior gastric artery, were $32.50 \%$ versus $100 \%, 16.25 \%$ versus $87.50 \%$, and $3.75 \%$ versus $25.00 \%$, respectively. According to Hiatt's classification, 75 out of 240 cases were abnormal types, among which we found Type II in 30 cases, Type III in 33 cases, Type IV in three cases, Type V in six cases, and Type VI in only three cases. There was no significant difference for total and every single variation type, between our group and Hiatt's group $(P>0.05)$.

Conclusion: The 256-slice spiral CT angiography can be regarded as an effective and accurate diagnostic modality for preoperative assessing anatomical arterial variations in gastric cancer; MIP was superior to VR at identifying variations of some small artery, whereas VR was better than MIP at showing anatomical arterial variations due to its three-dimensional effect.

Keywords: gastric cancer, artery, angiography, tomography, spiral computed

\section{Introduction}

According to the National Cancer Registry Center of China, gastric cancer is the second most common cancer and the third leading cause of cancer mortality in 2012. Surgical treatment plays an indispensable role in the treatment of patients with gastric cancer. Today, one of the latest developments in the treatment of gastric cancer involves the use of multidisciplinary treatment involving surgical operation. While treating these patients, standard D2 lymphatic radical dissection surgery should be combined with other therapies such as perioperative chemotherapy or radiochemotherapy. N2 regional lymph nodes are located along the main arteries of the stomach. However, perigastric arteries are characterized by several anatomical variations and originations. ${ }^{1-7}$ These anatomical variations escalate the complexities and difficulties of surgical operation. 
Therefore, it is mandatory for surgeons to perform preoperative assessment of the anatomical variations and origins before conducting surgical treatment on gastric cancer patients. It is useful for surgeons to get a clear idea about the patient's gastric cancer condition based on the anatomical variations, which helps them to decide on an individualized and comprehensive surgical operation scheme. In addition, it can also minimize the probability of developing unnecessary and unexpected injuries for patients with gastric cancer. ${ }^{8}$ In this way, the operation time can be shortened effectively. Furthermore, postoperative complications can be reduced through effective utilization of proper medical resources.

In previous research studies, surgeons mainly conducted multislice computed tomography (CT) such as 16 -slice ${ }^{9}$ or 64-slice ${ }^{10,11}$ CT angiography to preoperatively evaluate the anatomy of gastric arteries. Such a kind of preoperative evaluation was conducted in patients with gastric cancer before performing laparoscopy-assisted gastrectomy and open operations. ${ }^{10-16}$ In a previous study conducted by Sun et al, ${ }^{17}$ it was reported that 256-slice spiral CT angiography was useful in preoperatively assessing the mesenteric vascular anatomy of colon cancer patients. Apart from the aforementioned cases, the 256-slice spiral CT angiography was routinely used in the diagnosis and therapy of cardiac diseases, ${ }^{18-21}$ and it played an important role in the evaluation of coronary arteries. ${ }^{22-24}$ However, application of 256-slice spiral CT angiography in gastric cancer is not yet known. The 256-slice spiral CT is an advanced radiologic method that has a higher resolution in time and space than others. In this study, our ultimate goal was to extend the utilization of 256-slice spiral CT angiography for the preoperative evaluation of surrounding arteries in gastric cancer.

\section{Materials and methods Ethical approval}

Ethical approval was given by the Medical Ethics Committee of Guangdong General Hospital. Written informed consent from patients were obtained.

\section{Patients}

In the period extending from June 2012 to March 2013, we analyzed 80 gastric cancer patients diagnosed by endoscopic biopsy in Guangdong General Hospital. These patients underwent plain and enhanced CT scan that was followed by surgical treatment. In this study, we investigated 53 male and 27 female patients whose ages ranged between 28 and 83 years (mean age 57.3 years). Among the 80 patients, there were 32 patients whose tumors were located in the gastric antrum. The tumors of 25 patients were located in the gastric corpus, while 15 patients had tumors in the cardiac part and fundus.

\section{The 256-slice spiral CT angiography protocol}

CT scan images were obtained using a 256-slice spiral CT (PHILIPS Brilliance iCT from Royal Dutch Philips Electronics Ltd., Amsterdam, the Netherlands). After careful preparation of patients for CT scan, the following scanning parameters were used: a tube current of $400 \mathrm{~mA}$, voltage of $120 \mathrm{kV}$, scan time of 0.27 seconds per rotation, detector configuration of $0.625 \mathrm{~mm} \times 256$, and pitch of 0.938. Arterial and portal venous phase CT images were obtained after the intravenous injection of $1.5 \mathrm{~mL} / \mathrm{kg}$ of $300 \mathrm{mg} \mathrm{I} / \mathrm{mL}$ nonionic contrast material at a flow rate of $3 \mathrm{~mL} / \mathrm{sec}$. The thicknesses of precontrast phase, arterial phase, and portal phase were all set to $2.5 \mathrm{~mm}$. Portal venous phase scanning was performed 70 seconds after the start of bolus injection.

\section{Image analysis}

The original CT angiography data and images were transmitted to computer workstation (PHILIPS Brilliance iCT from Royal Dutch Philips Electronics Ltd.) in our hospital. Then, two senior radiologists reconstructed the perigastric CT angiograms by using volume rendering (VR) and maximum intensity projection (MIP) techniques. Thereafter, two senior surgeons observed and analyzed variant anatomical patterns of perigastric arteries through reconstructed angiograms. These preoperative findings were then compared with the vascular anatomy identified during the surgical operation conducted by the same surgical team. The differences of VR and MIP in reconstructing arteries were analyzed and the accuracy of 256-slice spiral CT angiography was estimated by using the surgical findings that were regarded as the standard of reference. According to Hiatt's ${ }^{1}$ arterial variations, the variant anatomical patterns were artificially divided into six types. The six types are

- Type I was defined as a normal pattern; the common hepatic artery (CHA) arises from the celiac axis (CA) to form the gastroduodenal and proper hepatic arteries, and the proper hepatic artery is divided distally into right and left branches.

- Type II: a replaced or accessory left hepatic artery arises from the left gastric artery (LGA).

- Type III: a replaced or accessory right hepatic artery. 
- Type IV: in this double-replaced pattern, the right hepatic artery arises from the superior mesenteric artery, and the left hepatic artery was a branch of the LGA.

- Type V: the entire CHA originates as a branch of the superior mesenteric artery.

- Type VI: the CHA directly originates from the aorta. ${ }^{1}$

\section{Statistical analysis}

Statistical analysis was performed by using $\chi^{2}$ test (Pearson's chi-squared test) for different variant anatomical patterns, whereas Mann-Whiney $U$-test was used for comparing the arterial variations between our group and Hiatt's group. $P$-value $<0.05$ was considered as a statistically significant difference. The SPSS 13.0 software (IBM Corporation, Armonk, NY, USA) was used in statistical analysis.

\section{Results}

As listed in Table 1, the anatomical angiograms of all 80 patients were obtained at the PHILIPS Brilliance iCT workstation by using VR and MIP reconstruction techniques.

Both VR and MIP techniques were used to reconstruct the perigastric arteries. Specifically, the reconstruction imaging rates for arteries of $\mathrm{CA}, \mathrm{CHA}$, and splenic artery were all $100 \%(80 / 80)$ by using VR and MIP. The imaging rates of MIP were $100 \%$ (80/80) for LGA, right gastric artery (RGA), left and right gatroepiploic artery, and gastroduodenal artery (GDA). For LGA, RGA, and GDA, the imaging rates of VR were 98.75\% (79/80), 91.25\% (73/80), and 95\% (76/80), respectively. VR imaging rates were inferior to MIP for arteries around the greater curvature and fundus, such as left gastroepiploic artery, short gastric artery, and posterior gastric artery, the respective rates of which were $32.50 \%$ (34/80) versus $100 \%(80 / 80), 16.25 \%(13 / 80)$ versus $87.50 \%$ $(70 / 80)$, and $3.75 \%(3 / 80)$ versus $25.00 \%(20 / 80)$. For these three arteries, the difference between VR and MIP was statistically significant $\left(\chi^{2}=6.857, P=0.32<0.05\right)$. Figure 1 , depicts the comparison between VR (Figure 1A and B) and MIP (Figure 1C and D) reconstruction for perigastric arteries.

As listed in Table 2, we compared various arterial variations of our group with some research related on this topic. According to the Hiatt's arterial variations, 55 cases in our group were regarded as normal pattern (Type I) and 25 cases were abnormal types, and the variation rate was $31.25 \%$. Among the abnormal cases, there were 10 cases whose left hepatic artery originated from the LGA (Type II, 12.50\%). There were eleven cases whose right hepatic arteries arose from the superior mesenteric artery (Type III, 13.75\%), while there was one case where the left hepatic artery originated from the LGA and right hepatic artery originated from the superior mesenteric artery (coexisting; Type IV, 1.25\%). There were two cases where the CHA arose from the superior mesenteric artery (Type V, 2.50\%). There was only one case whose CHA originated from the abdominal aorta (Type VI, 1.25\%).

Some rare anatomical variations were observed in our study and are shown in Figures 2-4. As shown in Figure 2A and $\mathrm{B}$, hepatic artery arose from mesenteric artery and could be regarded as Type $\mathrm{V}$. In Figure $3 \mathrm{~A}$ and $\mathrm{B}$, the right hepatic artery originated from the GDA and could be classified as Type III. LGA, which arose directly from the aorta (Figure 4) was a Type VI.

In addition, we compared the arterial variations of our group with Hiatt's arterial variations, and these results are given in Table 3. Between our group and Hiatt's arterial variations, the differences for every single variation type ranging from Type I to VI were not statistically significant $(P>0.05)$ as per $\chi^{2}$ test.

\section{Discussion}

Gastric cancer is a major public health issue as it is the fourth most common cancer and the second leading cause of cancer death in the world. However, the incidence and mortality of gastric cancer have fallen over the past several decades. ${ }^{25}$ Nowadays, multidisciplinary treatment, which is mainly based on surgery, is the conventional approach for treating gastric cancer patients. In the surgical operation, the main process involves radical dissection of the local regional lymph nodes distributed along the arteries. Thus, the precise assessment of arterial anatomy before operation becomes increasingly crucial for developing a surgical strategy. With the invention of a specialized technique termed "multislice spiral computed tomography angiography", we can

Table I Comparison between VR and MIP for reconstruction of perigastric arteries (case)

\begin{tabular}{lllllllllll}
\hline Technique & CA & CHA & SA & LGA & RGA & LGeA & RGeA & GDA & SGA & PGA \\
\hline VR & 80 & 80 & 80 & 79 & 73 & 34 & 76 & 76 & 13 & 3 \\
MIP & 80 & 80 & 80 & 80 & 80 & 80 & 80 & 80 & 70 & 20 \\
\hline
\end{tabular}

Abbreviations: VR, volume rendering; MIP, maximum intensity projection; CA, celiac axis; CHA, common hepatic artery; SA, splenic artery; LGA, left gastric artery; RGA, right gastric artery; LGeA, left gatroepiploic artery; RGeA, right gatroepiploic artery; GDA, gastroduodenal artery; SGA, short gastric artery; PGA, posterior gastric artery. 

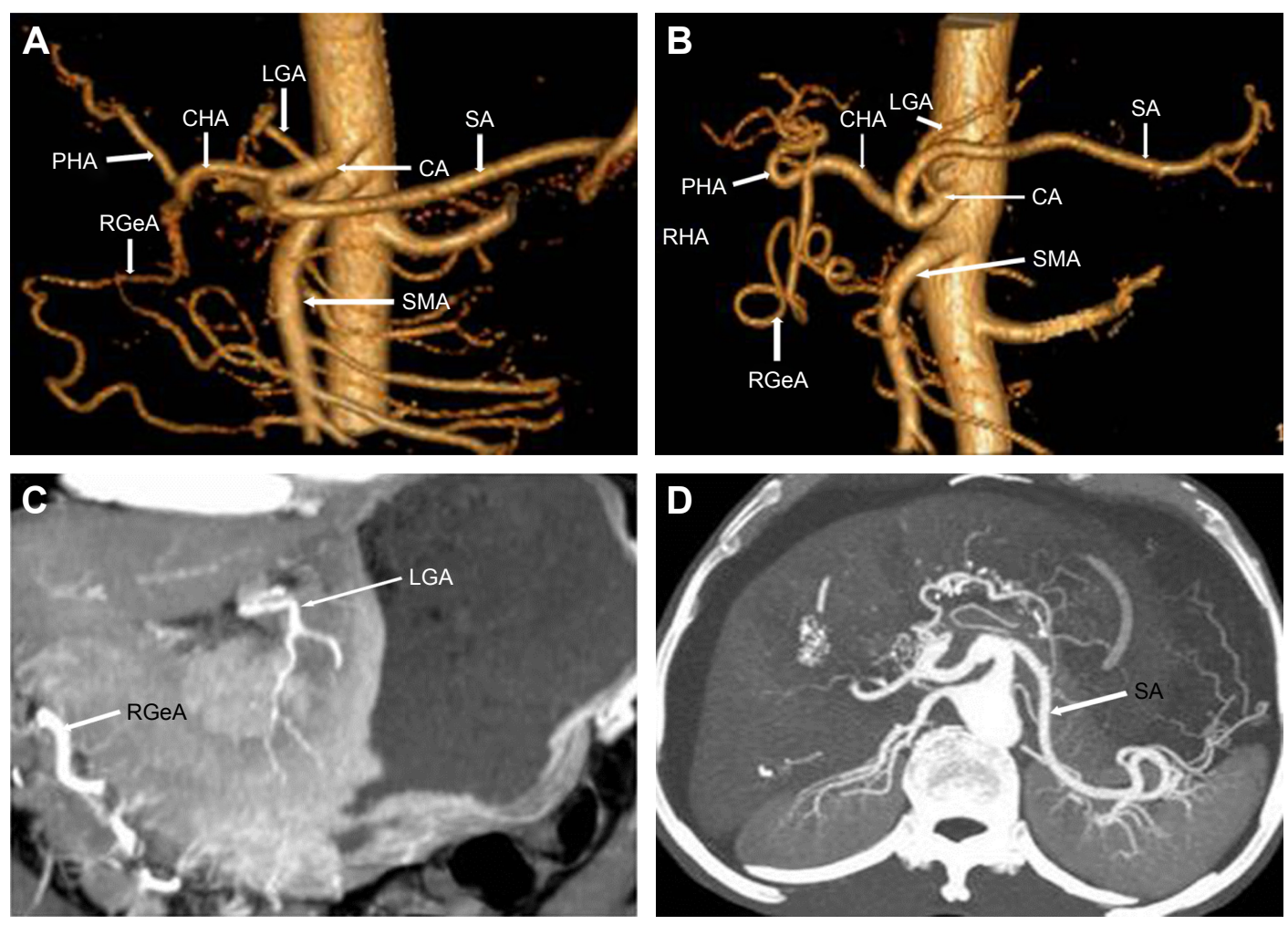

Figure I VR and MIP reconstruction techniques for perigastric arteries.

Notes: (A) VR reconstruction technique for perigastric arteries. The figure shows the gastric arteries of a 66-year-old man diagnosed with gastric cancer of the gastric corpus. (B) VR reconstruction technique for perigastric arteries. The representative figure is from a 58-year-old man diagnosed with early gastric cancer located at the cardia of stomach. (C) MIP reconstruction technique for perigastric arteries from the same patient in (A). (D) MIP reconstruction technique for perigastric arteries from the same patient in (B).

Abbreviations: CA, celiac axis; CHA, common hepatic artery; LGA, left gastric artery; SA, splenic artery; PHA, proper hepatic artery; SMA, superior mesenteric artery; RGeA, right gastroepiploic artery; RHA, right hepatic artery; RGeA, right gastroepiploic artery; VR, volume rendering; MIP, maximum intensity projection.

now obtain highly accurate and detailed information. This technique is characterized by high time and spatial resolution on perigastric arterial anatomy. Numerous research studies have reported that multislice spiral CT angiography was beneficial in identifying the location of different arteries prior to performing laparoscopy-assisted gastrectomy as well as open operations. ${ }^{9-15}$ This study was conducted to determine how effectively a recently developed 256-slice

Table 2 Comparison for perigastric arterial types by angiography (\%)

\begin{tabular}{lllll}
\hline $\begin{array}{l}\text { Variation } \\
\text { type }\end{array}$ & $\begin{array}{l}\text { Hiatt's } \\
(\mathbf{n}=1, \mathbf{0 0 0})\end{array}$ & $\begin{array}{l}\text { Michels' } \\
(\mathbf{n}=1 \mathbf{9 6})\end{array}$ & $\begin{array}{l}\text { Kemeny's } \\
(\mathbf{n}=\mathbf{1 0 0})\end{array}$ & $\begin{array}{l}\text { Our group } \\
(\mathbf{n}=\mathbf{8 0})\end{array}$ \\
\hline I & 75.70 & 56.12 & 59.00 & 68.75 \\
II & 9.70 & 18.37 & 17.00 & 12.50 \\
III & 10.60 & 18.37 & 18.00 & 13.75 \\
IV & 2.30 & 4.08 & 2.00 & 1.25 \\
V & 1.50 & 2.55 & 3.00 & 2.50 \\
VI & 0.20 & 0.51 & 1.00 & 1.25 \\
\hline
\end{tabular}

Note: Arterial variation types were referenced by Hiatt's variation type and the variations between our group's and Hiatt's, Michels', and Kemeny's groups were compared. spiral CT angiography can evaluate surrounding arteries in gastric cancer.

VR, MIP, multiplanar reconstructions, curved planar reformations, and shaded surface display were some of the methods of angiogram construction. VR and MIP reconstruction techniques were mainly used in evaluating perigastric arteries. VR technique, which requires every sample value to be mapped to opacity and a color, is very useful in revealing the vascular patterns and anatomical variations due to threedimensional and intuitive effect. Unlike VR, MIP picks out and projects only the voxels with maximum intensity, which fall in the way of parallel rays traced from the viewpoint to the plane of projection. So, the images constructed by MIP technique are not very capable of revealing the adjacent tissues. As listed in Table 1, clear angiograms were obtained by both VR and MIP reconstruction techniques for some well-known arteries like CA, LGA and RGA, CHA, and splenic artery. However, it is helpful to use MIP technique in reconstructing some small or tiny arteries, such as short gastric artery and posterior gastric artery compared with VR. 

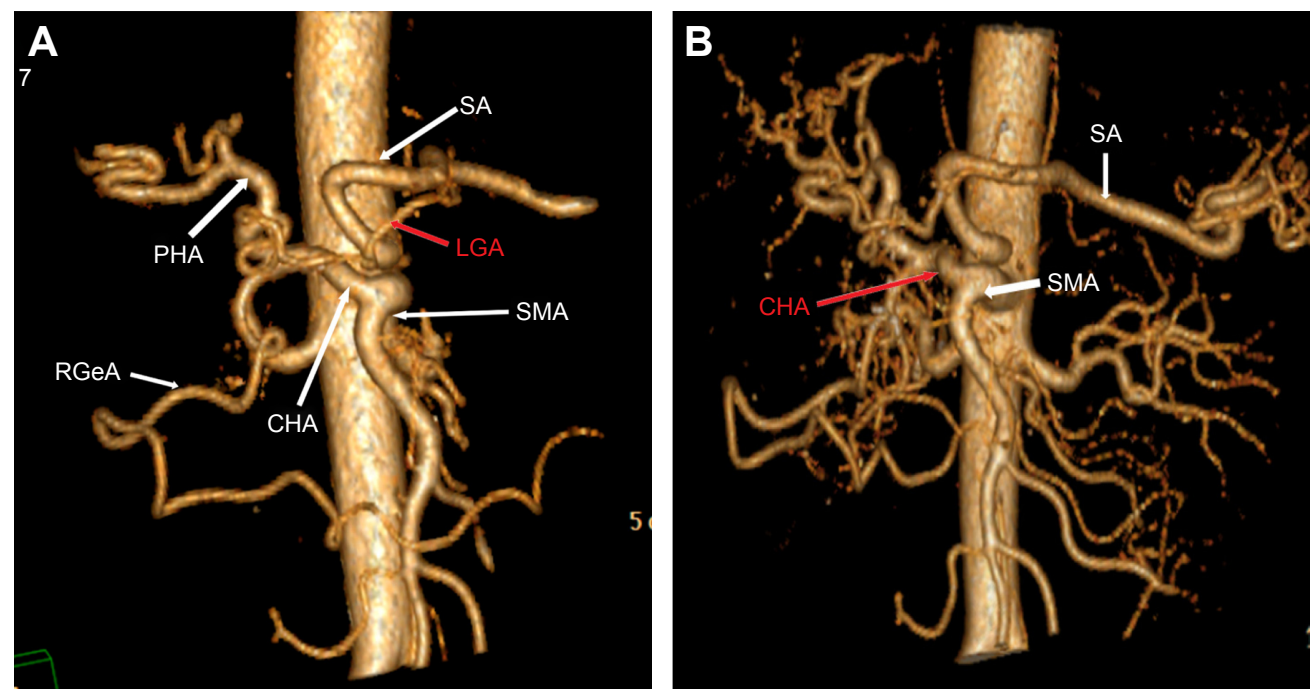

Figure 2 Three-dimensional reconstruction of gastric vessel anatomy and rare variations.

Notes: (A) CHA originating from superior mesenteric artery. This is an image of the gastric arteries of a 69-year-old woman diagnosed with gastric cancer located at gastric antrum. Hepatic artery arising from mesenteric artery could be regarded as arterial variation Type II. (B) CHA originating from superior mesenteric artery of a 73-year-old man diagnosed with gastric cancer of gastric antrum. Hepatic artery arising from mesenteric artery could be regarded as arterial variation Type II. The variant vessles LGA in (A), and CHA in (B) are written in red.

Abbreviations: CHA, common hepatic artery; SA, splenic artery; LGA, left gastric artery; RGeA, right gatroepiploic artery; SMA, superior mesenteric artery; PHA, proper hepatic artery.

It should be noted that neither VR nor MIP was ideal for the reconstruction of the posterior gastric artery in our study. In our previous work, ${ }^{26}$ it was reported that the occurrence probability of posterior gastric artery was approximately $28 \%$, and it was generally tiny. Therefore, the low reconstruction rate $(25 \%)$ in our group was associated with the low occurrence probability of posterior gastric artery. MIP played a much more important role in reconstructing some tiny vessels, especially those that were supplying gastric cancer. Furthermore, it was beneficial to illustrate the comprehensive invasion by the neoplasm for a certain artery. MIP was chosen as the technique of operation under the condition that the target vessel and the peripheral soft tissues were similar when enhancement was performed. On the contrary, VR was applied when obvious difference existed.

There are numerous arterial variant patterns and various anatomical originations around the stomach. Some research papers, which were related to this topic, stated that the variation rate varied from $20 \%$ to $45 \% .^{1-7}$ The abnormal variation rate in our group was $31.25 \%$, and there was no significant difference between our group and Hiatt's arterial variations for every single variation type ranging
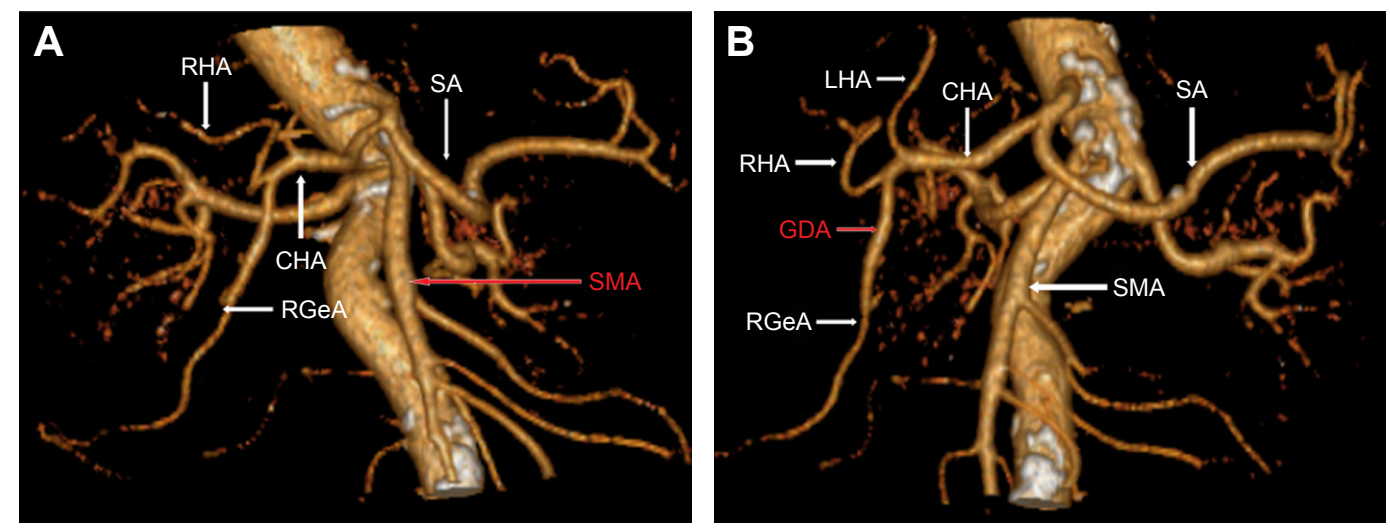

Figure 3 Right hepatic artery deriving from GDA.

Notes: (A) The image shows the gastric arteries of a 58-year-old man diagnosed with early gastric cancer at the gastric corpus. Right hepatic artery was originating from GDA, which could to be classified as Type III. (B) Right hepatic artery deriving from GDA. The figure is from a 6I-year-old man diagnosed with gastric cancer located at gastric antrum. Right hepatic artery was originating from GDA, which could be classified as Type III. The variant vessles SMA in (A), and GDA in (B) are written in red. Abbreviations: CHA, common hepatic artery; SA, splenic artery; RHA, right hepatic artery; SMA, superior mesenteric artery; RGeA, right gastroepiploic artery; GDA, gastroduodenal artery; LHA, left hepatic artery. 


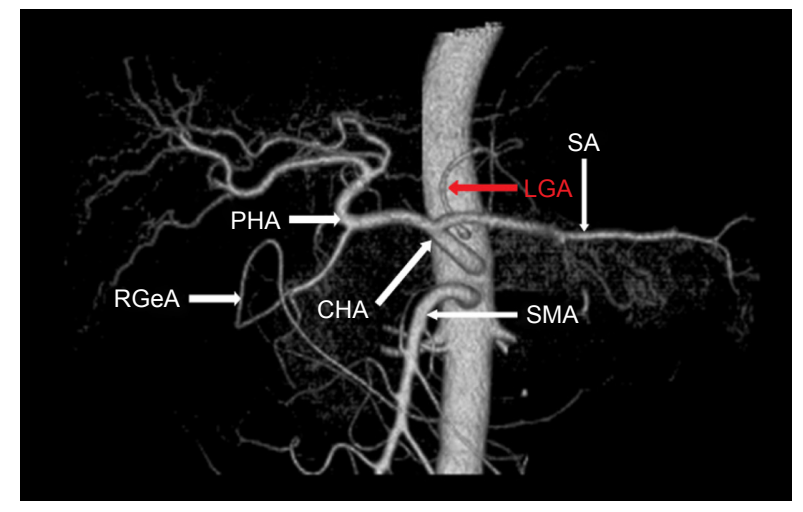

Figure 4 LGA originating from aorta.

Notes: This was from a 77-year-old man diagnosed with gastric cancer located at gastric antrum. LGA (in red) and superior mesenteric artery were directly originating from aorta.

Abbreviations: CHA, common hepatic artery; SA, splenic artery; SMA, superior mesenteric artery; RGeA, right gastroepiploic artery; PHA, proper hepatic artery; LGA, left gastric artery.

from I to VI $(P>0.05)$. As shown in Figures $2-4$, we also found some anatomical variations around the stomach by VR reconstruction technique. If these vascular variations had not been recognized by surgeons before operation, there would have been an increased risk of bleeding due to unexpected vascular injures. Consequently, surgeons would have to deal with unnecessary complications during operation. In addition, surgeons found it difficult to accomplish the D2 lymphatic dissection in radical operation, which could not conform to tumor-free principle. Thus, the recurrence risk could be probably increased, whereas the disease-free survival and overall survival could be hardly improved effectively for patients with gastric cancer. According to Japanese gastric cancer association, the seventh lymph nodes, which are located along the LGA, are supposed to be dissected in surgical treatment for gastric cancer. ${ }^{27}$ As shown in Figure 4, we were confronted with the following anatomical variation: the LGA was originating directly from the aorta and this situation had not been observed before operation. This kind of anatomical variation can undoubtedly increase the

Table 3 Comparison of arterial variations between our group and Hiatt's (case)

\begin{tabular}{lllll}
\hline $\begin{array}{l}\text { Hiatt } \\
\text { type }\end{array}$ & $\begin{array}{l}\text { Hiatt } \\
(\mathbf{n}=\mathbf{I}, \mathbf{0 0 0})\end{array}$ & $\begin{array}{l}\text { Our group } \\
(\mathbf{n}=\mathbf{8 0})\end{array}$ & $\chi^{2}$ value & P-value \\
\hline I & 757 & 55 & 1.918 & 0.166 \\
II & 97 & 10 & $0.65 \mathrm{I}$ & 0.420 \\
III & I06 & II & 0.272 & 0.602 \\
IV & 23 & I & $0.048^{\mathrm{a}}$ & 0.827 \\
V & I5 & 2 & $0.05 \mathrm{I}^{\mathrm{a}}$ & 0.822 \\
VI & 2 & $\mathrm{I}$ & $0.376^{\mathrm{a}}$ & 0.540 \\
\hline
\end{tabular}

Notes: aResult of continuity correction. The differences for every single variation type ranging from Type I to VI, between our group and Hiatt's arterial variations. difficulty for lymphatic dissection. In other words, we could not ignore this group of lymph nodes during the whole operation process. The prognosis of patients would be certainly affected under this condition.

\section{Conclusion}

The 256-slice spiral CT angiography can be considered as an effective and accurate diagnostic modality for preoperative assessment of anatomical arterial variations in gastric cancer. MIP was superior to VR in revealing some small arteries, whereas VR was better than MIP in showing the anatomical arterial variations due to its three-dimensional effect. The results of our study indicated that 256-slice spiral CT angiography can provide beneficial guidance in making appropriate surgical strategy of gastric cancer. Further research is still required to explore new approaches that help in evaluating the anatomical arteries.

\section{Acknowledgments}

Thanks to Cao Xi-Da, Zhen Jun-Hui, and Wang Si-Yun from the Department of Radiology for the assistance they provided to get numerous CT dates together. This work was supported by Guangdong Provincial Natural Scientific Foundation (number S2011010001430), Guangdong Science and Technology Department Scientific Foundation (number 2010B060900064), Science Department of Guangzhou Scientific Foundation (number 2010Y1-C121), Yuexiu Science and Information Center of Guangzhou Scientific Foundation (number 2012-GX-046), and National Natural Scientific Foundation of China (number 81001112).

\section{Disclosure}

The authors report no conflicts of interests in this work.

\section{References}

1. Nakanishi R, Endo K, Yoshinaga K, et al. Unique variation of the hepatic artery identified on preoperative three-dimensional computed tomography angiography in surgery for gastric cancer: report of a case. Surg Today. 2010;40(10):967-971.

2. Song SY, Chung JW, Yin YH, et al. Celiac axis and common hepatic artery variations in 5002 patients: systematic analysis with spiral CT and DSA. Radiology. 2010;255(1):278-288.

3. Winston CB, Lee NA, Jarnagin WR, et al. CT angiography for delineation of celiac and superior mesenteric artery variants in patients undergoing hepatobiliary and pancreatic surgery. AJR Am J Roentgenol. 2007; 189(1):W13-W19.

4. Koops A, Wojciechowski B, Broering DC, Adam G, Krupski-Berdien G. Anatomic variations of the hepatic arteries in 604 selective celiac and superior mesenteric angiographies. Surg Radiol Anat. 2004;26(3): 239-244.

5. Hiatt JR, Gabbay J, Busuttil RW. Surgical anatomy of the hepatic arteries in 1000 cases. Ann Surg.1994;220(1):50-52.

6. Michels NA. Newer anatomy of the liver and its variant blood supply and collateral circulation. Am J Surg. 1966;112(3):337-347. 
7. Kemeny MM, Hogan JM, Goldberg DA, et al. Continuous hepatic artery infusion with an implantable pump: problems with hepatic artery anomalies. Surgery. 1986;99(4):501-504.

8. Xie E, Weng ZS, Wang XZ, Huang YK. Clinical significance of multi-slice spiral CT angiography in radical resection of gastric cancer. Zhonghua Wei Chang Wai Ke Za Zhi. 2011;14(1):31-33.

9. Kumano S, Tsuda T, Tanaka H, et al. Preoperative evaluation of perigastric vascular anatomy by 3-dimensional computed tomographic angiography using 16-channel multidetector-row computed tomography for laparoscopic gastrectomy in patients with early gastric cancer. J Comput Assist Tomogr. 2007;31(1):93-97.

10. Yao XQ, Lin F, Zhang ZL, et al. Preoperative evaluation of the 64-slice spiral CT three dimensional angiography for vascular invasion in gastric cancer. Zhonghua Wei Chang Wai Ke Za Zhi. 2008;11(5):440-443.

11. Li X, Chu J, Sun C, et al. Sixty-four-slice computed tomography angiography of perigastric veins with image fusion. J Comput Assist Tomogr. 2013;37(2):165-170.

12. Kawasaki K, Kanaji S, Kobayashi I, et al. Multidetector computed tomography for preoperative identification of left gastric vein location in patients with gastric cancer. Gastric Cancer. 2010;13(1):25-29.

13. Matsuki M, Matsuki M, Kani H, et al. Preoperative assessment of vascular anatomy around the stomach by $3 \mathrm{D}$ imaging using MDCT before laparoscopy-assisted gastrectomy. AJR Am J Roentgenol. 2004; 183(1):145-151.

14. Miyaki A, Imamura K, Kobayashi R, Takami M, Matsumoto J, Takada Y. Preoperative assessment of perigastric vascular anatomy by multidetector computed tomography angiogram for laparoscopy-assisted gastrectomy. Langenbecks Arch Surg. 2012;397(6):945-950.

15. Usui S, Hiranuma S, Ichikawa T, Maeda M, Kudo SE, Iwai T. Preoperative imaging of surrounding arteries by three-dimensional CT: is it useful for laparoscopic gastrectomy? Surg Laparosc Endosc Percutan Tech. 2005;15(2):61-65.

16. Kim HS, Han HY, Choi JA, et al. Preoperative evaluation of gastric cancer: value of spiral CT during gastric arteriography (CTGA). Abdom Imaging. 2001;26(2):123-130.

17. Sun HL, Wang W, Yao L, et al. Preoperative evaluation of mesenteric vascular anatomy using 256 multi-slice computed tomography before laparoscopic surgery. Zhonghua Wei Chang Wai Ke Za Zhi.2011;14(11): $855-858$
18. Dagvasumberel M, Shimabukuro M, Nishiuchi T, et al. Gender disparities in the association between epicardial adipose tissue volume and coronary atherosclerosis: a 3-dimensional cardiac computed tomography imaging study in Japanese subjects. Cardiovasc Diabetol. 2012;11:106.

19. Hou Y, Liu X, Xv S, Guo W, Guo Q. Comparisons of image quality and radiation dose between iterative reconstruction and filtered back projection reconstruction algorithms in 256-MDCT coronary angiography. AJR Am J Roentgenol. 2012;199(3):588-594.

20. Mor-Avi V, Lodato JA, Kachenoura N, et al. Quantitative threedimensional evaluation of myocardial perfusion during regadenoson stress using multidetector computed tomography. J Comput Assist Tomogr. 2012;36(4):443-449.

21. Li JL, Huang MP, Liang CH, et al. Individualized radiation dose control in 256-slice CT coronary angiography (CTCA) in retrospective ECGtriggered helical scans: using a measure of body size to adjust tube current selection. Eur J Radiol. 2012;81(11):3146-3153.

22. Segev OL, Gaspar T, Halon DA, et al. Image quality in obese patients undergoing 256-row computed tomography coronary angiography. Int J Cardiovasc Imaging. 2012;28(3):633-639.

23. Steen $\mathrm{H}$, André F, Korosoglou G, et al. In vitro evaluation of 56 coronary artery stents by 256-slice multi-detector coronary CT. Eur J Radiol. 2011;80(1):143-150.

24. Hosch W, Heye T, Schulz F, et al. Image quality and radiation dose in 256-slice cardiac computed tomography: comparison of prospective versus retrospective image acquisition protocols. Eur J Radiol. 2011;80(1):127-135.

25. Crew KD, Neugut AI. Epidemiology of gastric cancer. World J Gastroenterol. 2006;12(3):354-362.

26. Yao XQ, Lin F, Zhang ZL, et al. Preoperative evaluation of the 64-slice spiral CT three dimensional angiography for vascular invasion in gastric cancer. Chinese Journal of Gastrointestinal Surgery. 2008; 11(5):440-443. 1994;220(1):50-52.

27. Lee SL, Lee HH, Ko YH, et al. Relevance of hepatoduodenal ligament lymph nodes in resectional surgery for gastric cancer. Br J Surg. 2014;101(5):518-522.
OncoTargets and Therapy

\section{Publish your work in this journal}

OncoTargets and Therapy is an international, peer-reviewed, open access journal focusing on the pathological basis of all cancers, potential targets for therapy and treatment protocols employed to improve the management of cancer patients. The journal also focuses on the impact of management programs and new therapeutic agents and protocols on

\section{Dovepress}

patient perspectives such as quality of life, adherence and satisfaction. The manuscript management system is completely online and includes a very quick and fair peer-review system, which is all easy to use. Visit http://www.dovepress.com/testimonials.php to read real quotes from published authors. 